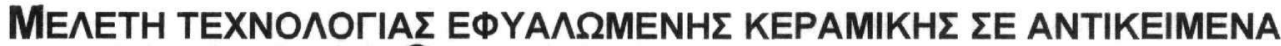

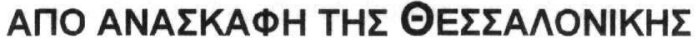

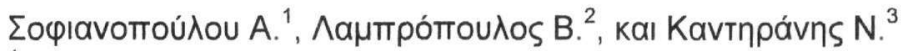

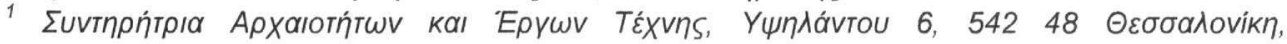 \\ alecasof@otenet.gr

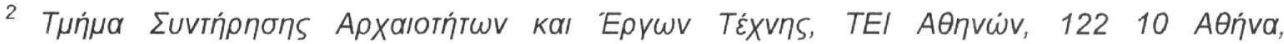 \\ blabro@teiath.gr

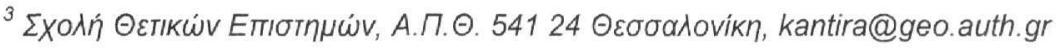

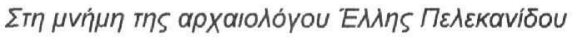

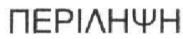

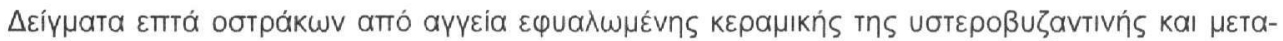

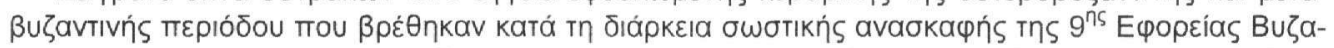

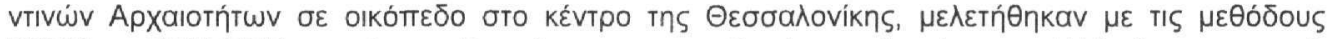

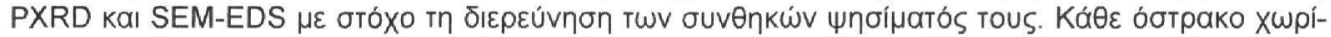

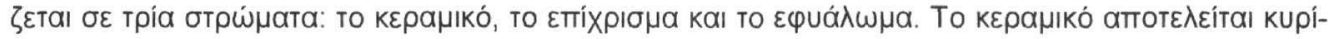

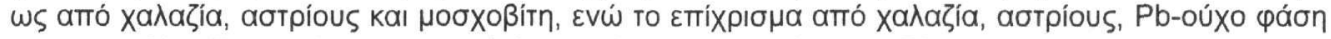

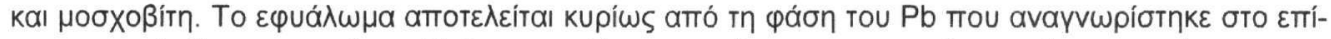

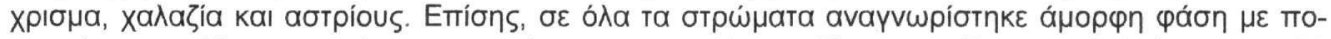

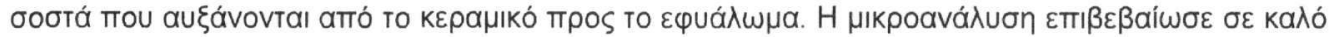

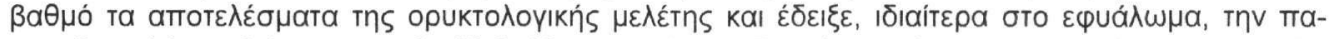

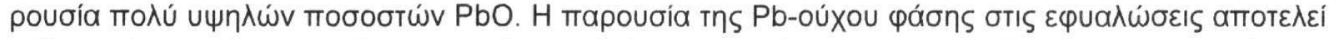

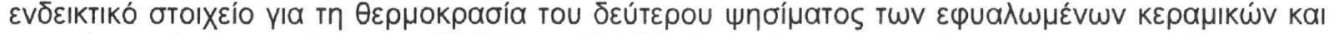

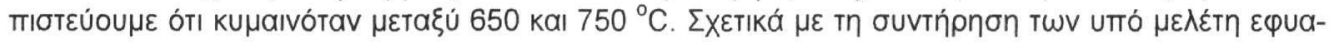

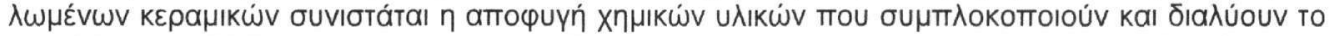

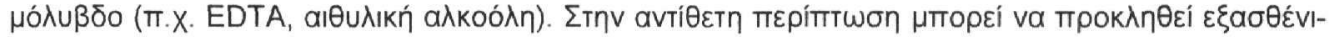

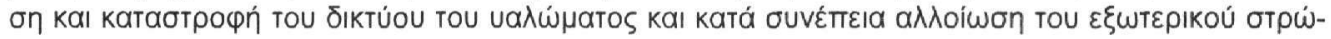

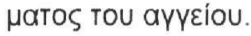

\section{$1 \mathrm{EI} \Sigma \mathrm{A} \Gamma \Omega \Gamma \mathrm{H}$}

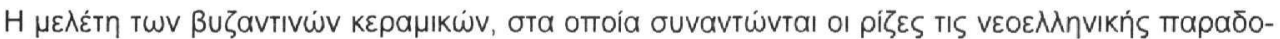

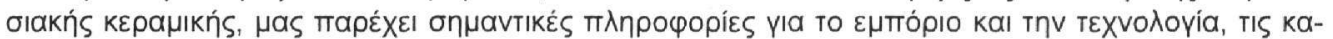

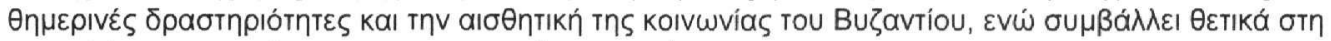

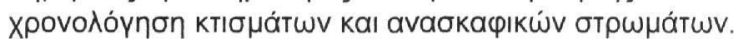

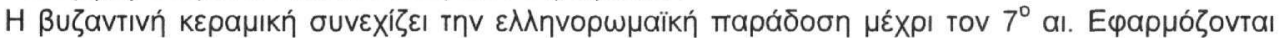

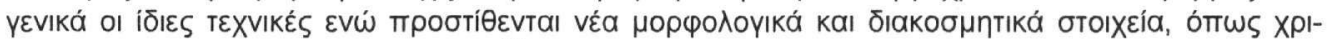

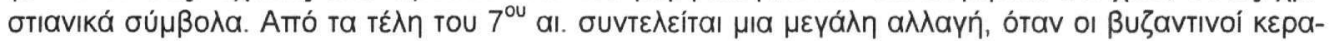

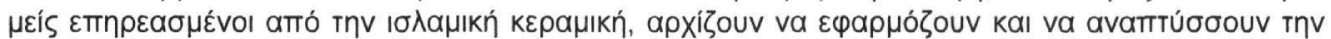

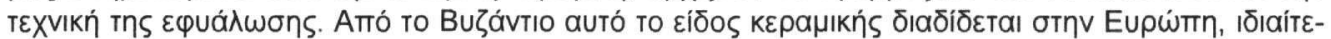

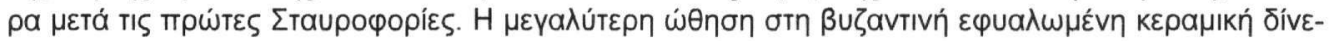

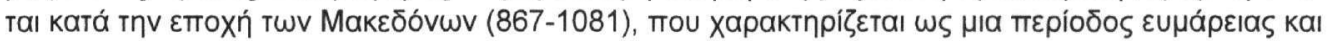

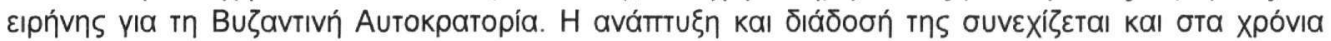

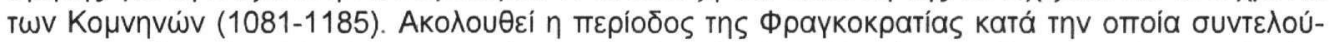
vтаı $\mu о \rho \varphi$

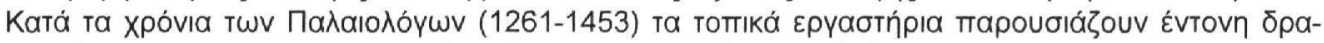

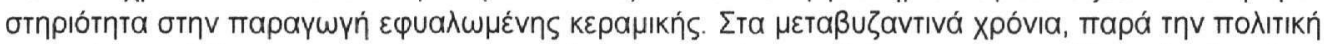

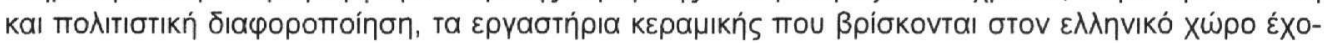




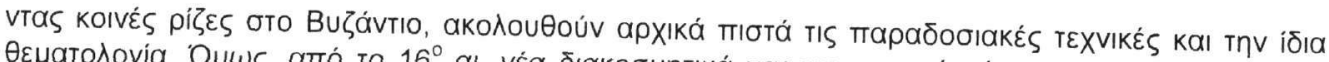

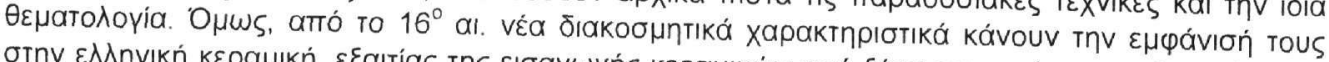

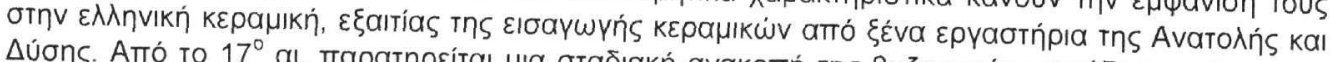

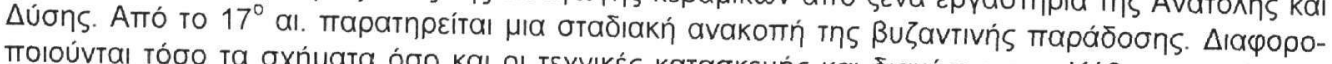

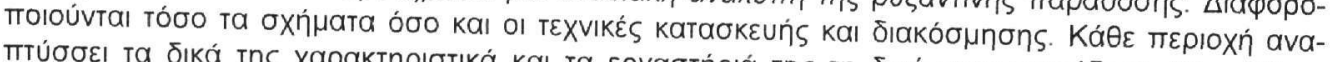

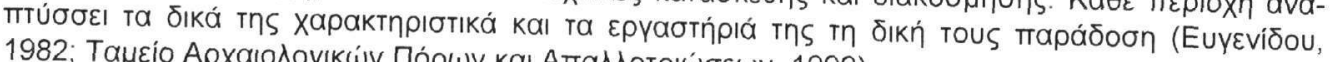

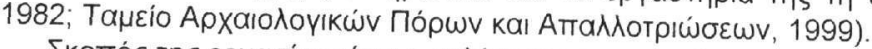

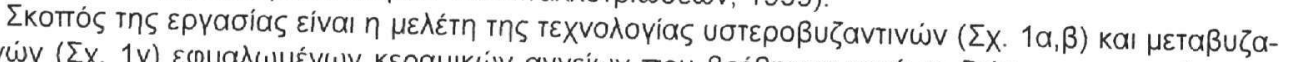

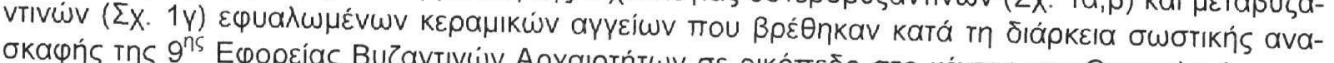

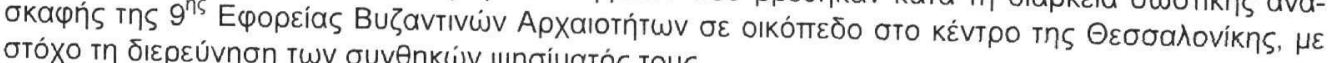

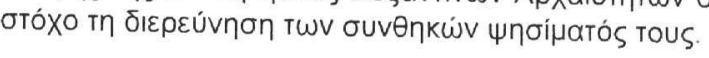

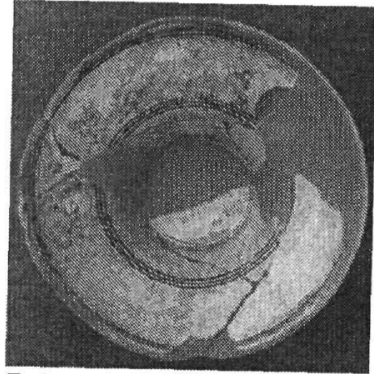

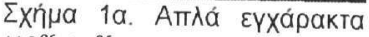
$\left(13^{o \zeta}-14^{\circ 5}\right.$ al. $)$

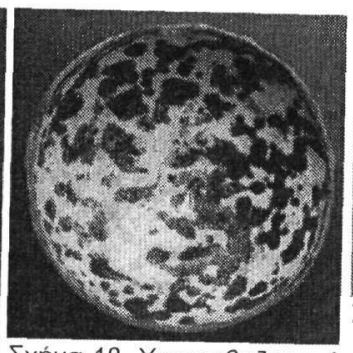

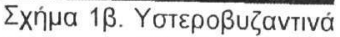

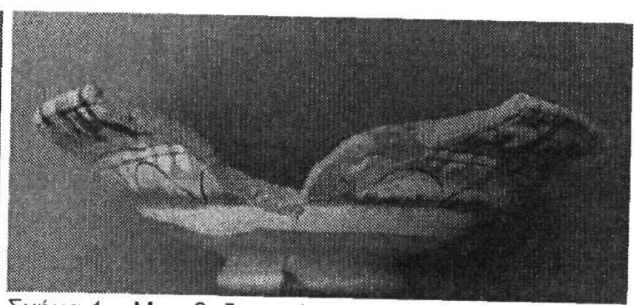

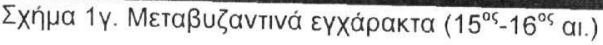

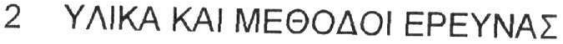

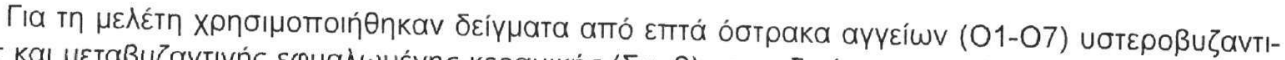

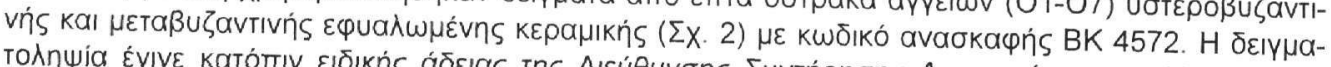

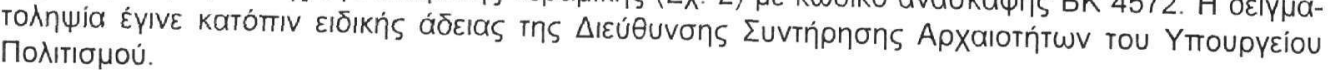

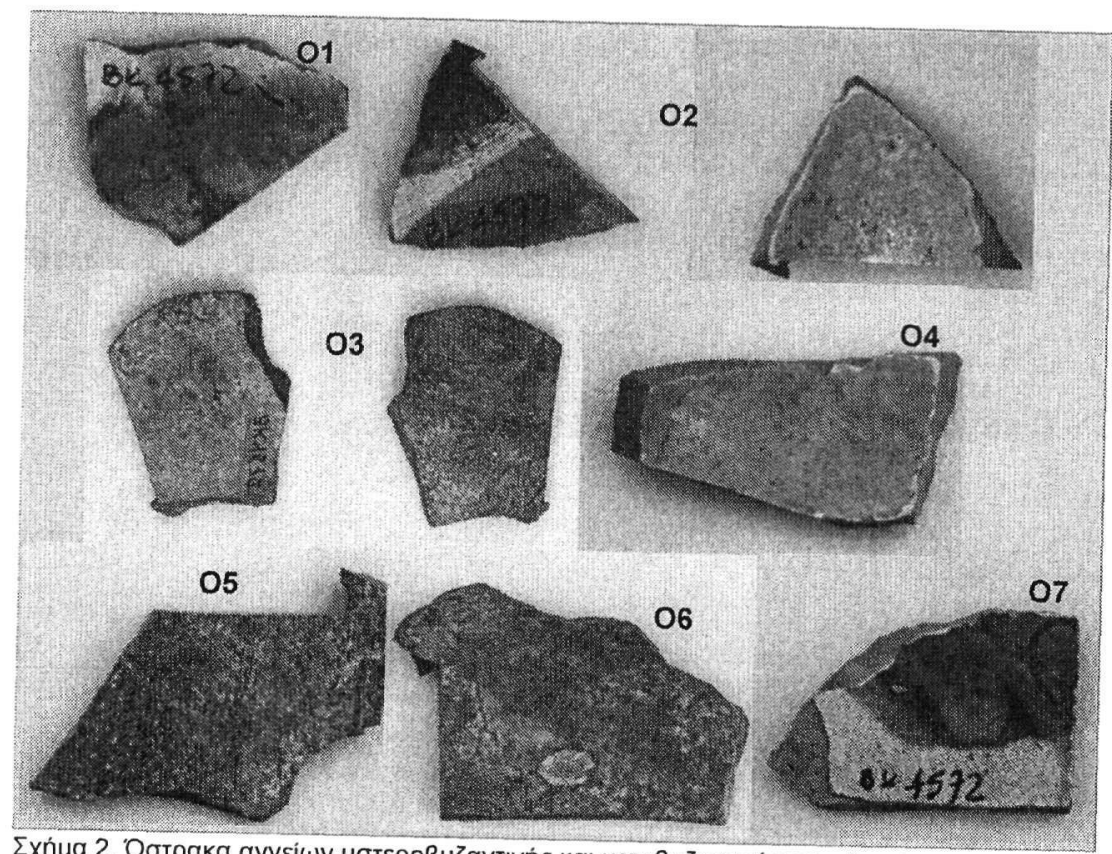

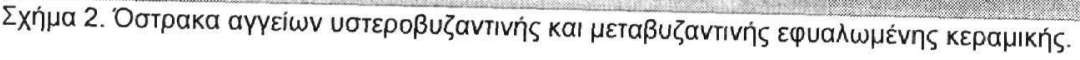




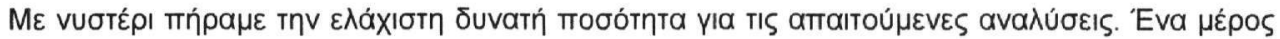

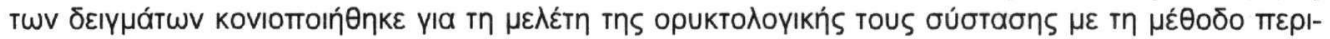

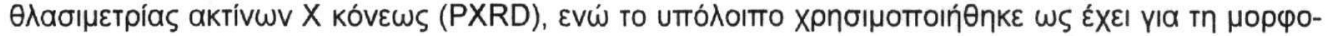

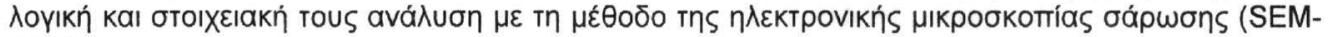
EDS).

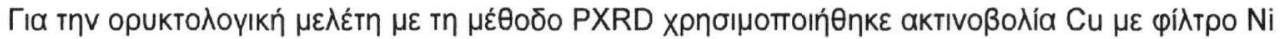

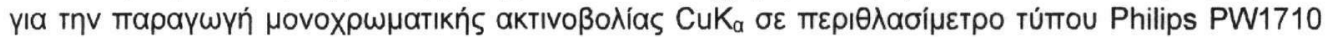

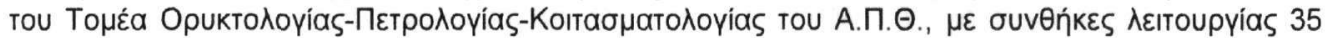

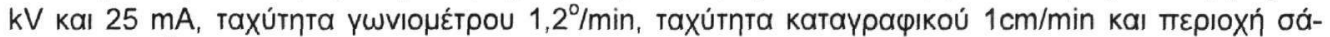

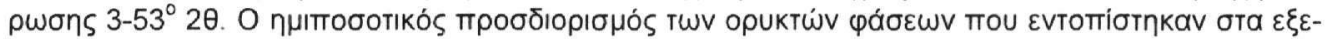

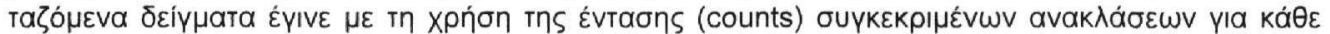

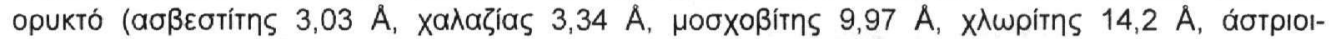

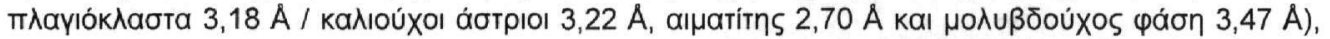

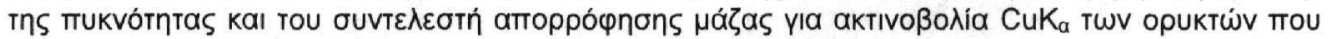

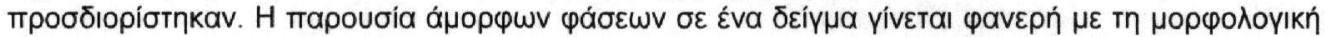

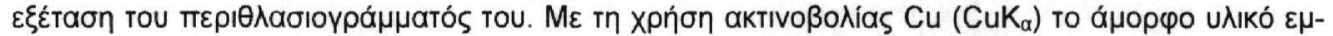

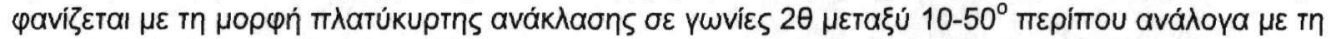

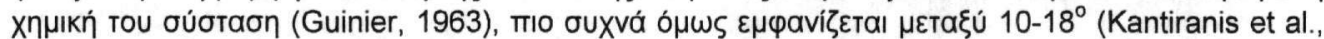

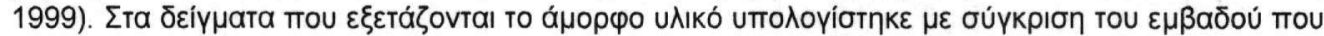

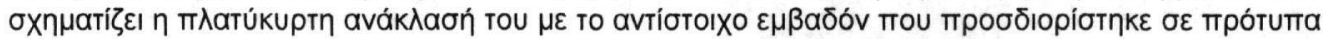

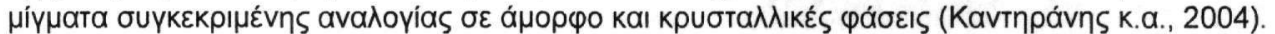

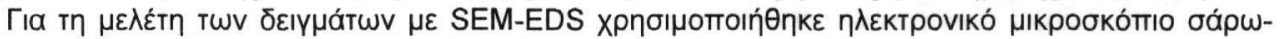

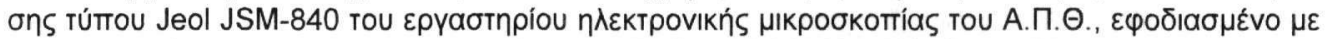

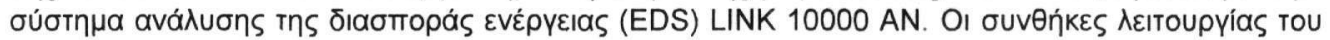

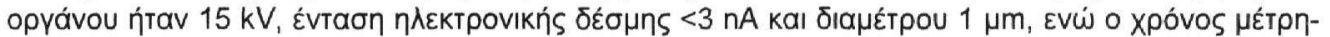

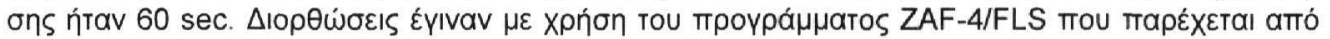
In LINK.

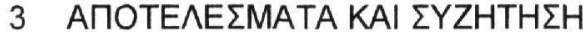

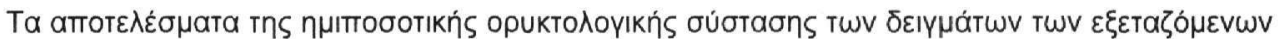

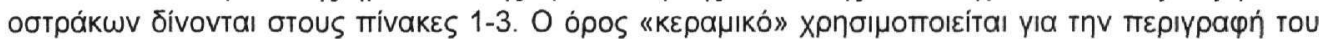

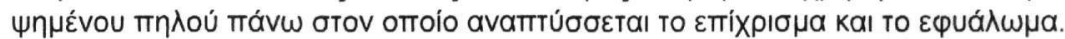

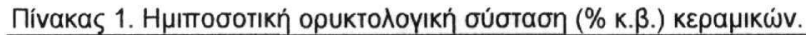

\begin{tabular}{|c|c|c|c|c|c|c|c|c|c|}
\hline Оотрако & $\mathbf{Q}$ & $F$ & M & C & $\mathrm{H}$ & Ch & A & 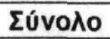 & Am \\
\hline 01 & 70 & 12 & 3 & 13 & - & - & - & 98 & 2 \\
\hline $\mathrm{O} 2$ & 63 & 11 & 10 & 12 & - & 2 & - & 98 & 2 \\
\hline O3 & 55 & 22 & 4 & 4 & 9 & 4 & - & 98 & 2 \\
\hline O4 & 67 & 20 & 10 & - & - & - & - & 97 & 3 \\
\hline O5 & 78 & 9 & 11 & - & - & - & - & 98 & 2 \\
\hline O6 & 59 & 16 & 11 & 9 & - & - & 2 & 97 & 3 \\
\hline 07 & 59 & 22 & 15 & - & - & - & - & 96 & 4 \\
\hline
\end{tabular}

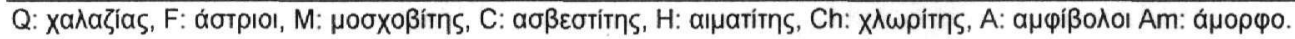

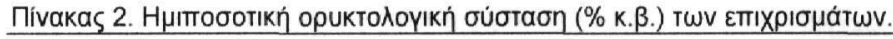

\begin{tabular}{|c|c|c|c|c|c|c|}
\hline Оотрако & $\mathbf{Q}$ & $\mathbf{F}$ & $M$ & $\mathbf{P b}$ & Eúvoגo & Am \\
\hline 01 & 74 & 11 & 4 & 4 & 93 & 7 \\
\hline O4 & 66 & 16 & 2 & 13 & 97 & 3 \\
\hline O6 & 74 & 16 & 3 & 3 & 96 & 4 \\
\hline O7 & 75 & 10 & 4 & 5 & 94 & 6 \\
\hline
\end{tabular}

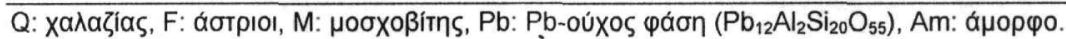

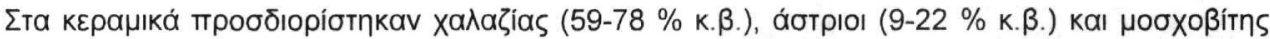

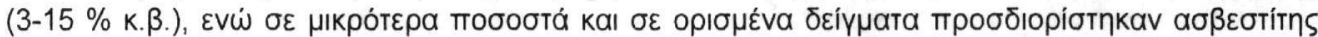

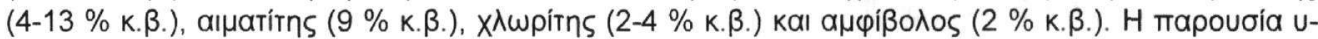




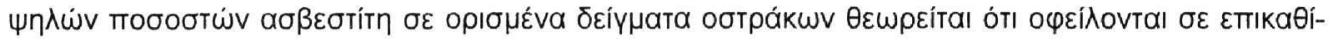

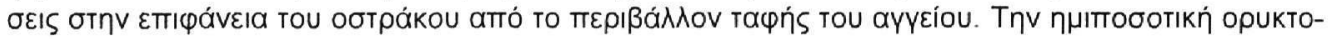

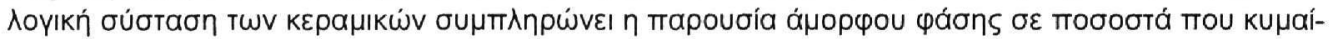

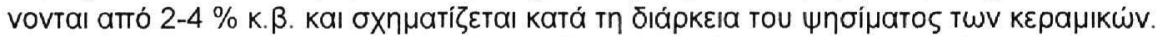

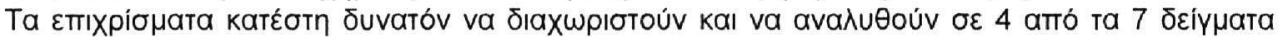

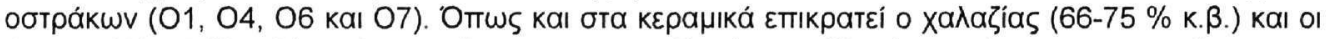

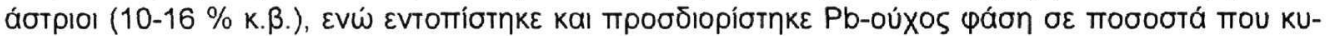

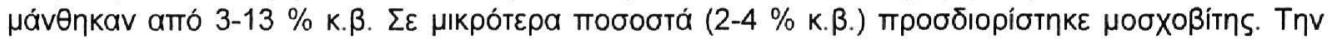

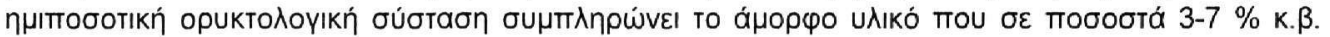

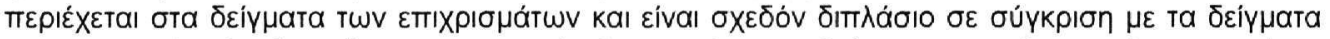

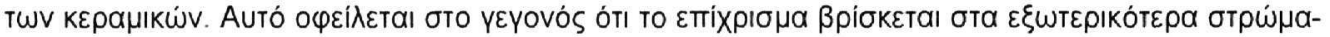

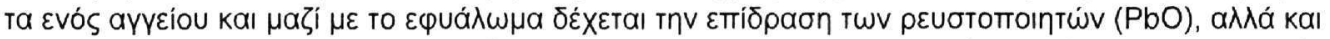

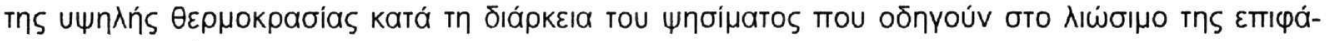
veıas tou aypziou.

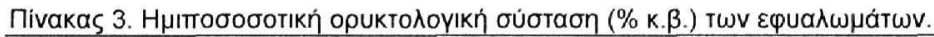

\begin{tabular}{|c|c|c|c|c|c|c|}
\hline Оотрако & $\mathbf{Q}$ & $\mathbf{F}$ & $M$ & $\mathrm{~Pb}$ & Eúvoגo & Am \\
\hline 01 & 46 & 25 & - & 10 & 81 & 19 \\
\hline $\mathrm{O} 2$ & 56 & 12 & 3 & 12 & 83 & 17 \\
\hline $\mathrm{O} 3$ & 61 & 15 & - & 10 & 86 & 14 \\
\hline O4 & 45 & 16 & - & 16 & 77 & 23 \\
\hline 05 & 54 & 19 & 3 & 9 & 85 & 15 \\
\hline O6 & 67 & 8 & 5 & 5 & 85 & 15 \\
\hline 07 & 60 & 13 & 3 & 7 & 83 & 17 \\
\hline
\end{tabular}

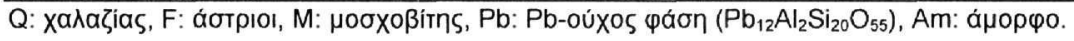

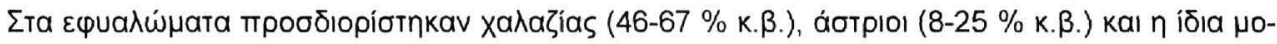

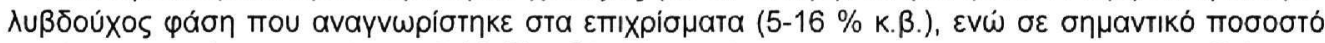

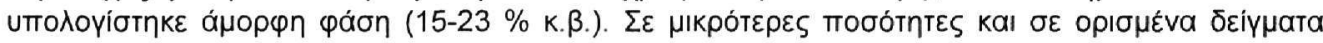

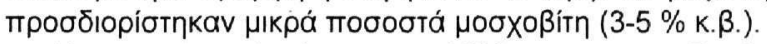

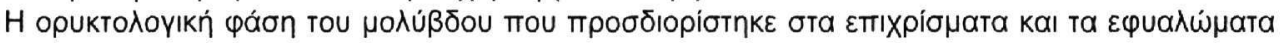

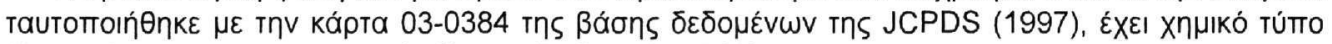

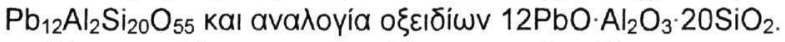

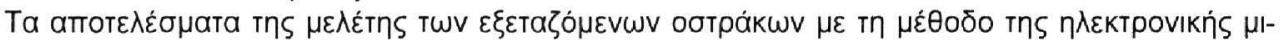

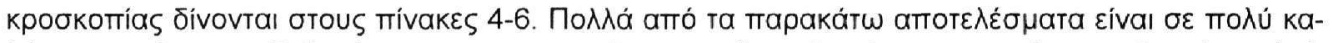

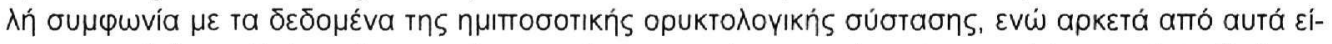

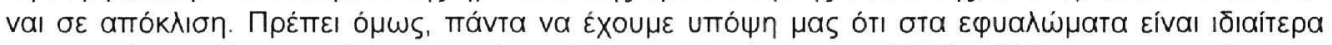

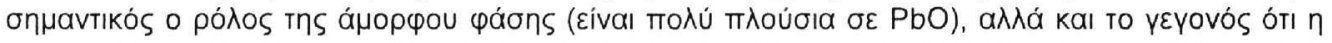

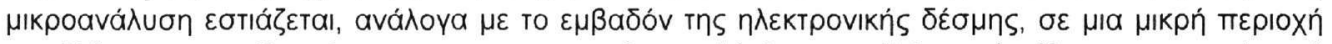

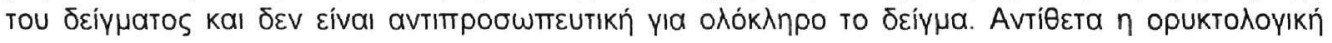

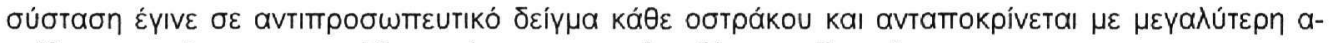

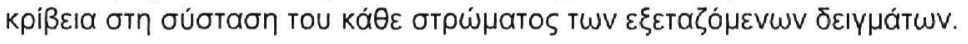

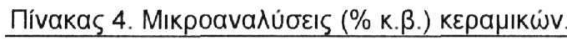

\begin{tabular}{|c|c|c|c|c|c|c|c|c|c|c|c|c|}
\hline Обтрако & $\mathrm{SiO}_{2}$ & $\mathrm{TiO}_{2}$ & $\mathrm{Al}_{2} \mathrm{O}_{3}$ & $\mathrm{Fe}_{2} \mathrm{O}_{3} \mathrm{~T}$ & MgO & $\mathrm{CaO}$ & $\mathrm{Na}_{2} \mathrm{O}$ & $\mathrm{K}_{2} \mathrm{O}$ & $\mathrm{P}_{2} \mathrm{O}_{5}$ & $\mathrm{PbO}$ & CuO & Eúvoגo \\
\hline 01 & 41,5 & - & 27,8 & 1,8 & - & 27,2 & 1,2 & 0,4 & - & - & - & 99,9 \\
\hline $\mathrm{O} 2$ & 75,9 & - & 20,4 & 0,6 & 0,2 & 0,2 & 0,8 & 0,7 & 1,0 & - & - & 99,8 \\
\hline $\mathrm{O} 3$ & 59,1 & - & 18,1 & 6,7 & 1,6 & 2,0 & 1,8 & 5,1 & - & 5,4 & - & 99,8 \\
\hline 04 & 35,1 & - & 21,7 & 25,5 & 6,8 & 1,2 & 1,7 & 2,4 & - & 5,5 & - & 99,9 \\
\hline O5 & 65,2 & - & 18,2 & 6,8 & 3,1 & 1,8 & 1,8 & 2,9 & - & - & - & 99,8 \\
\hline O6 & 35,4 & - & 24,3 & 23,5 & 11,3 & 1,0 & 1,2 & 1,5 & - & 1,7 & - & 99,9 \\
\hline $\mathrm{O} 7$ & 67,4 & - & 15,8 & 0,7 & - & 0,5 & 3,4 & 12,0 & - & - & - & 99,8 \\
\hline
\end{tabular}

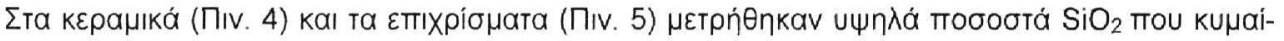

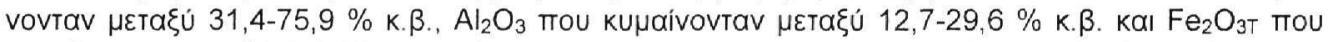

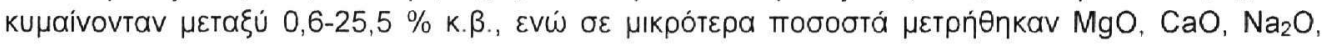




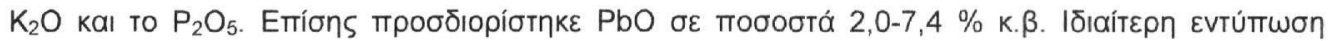

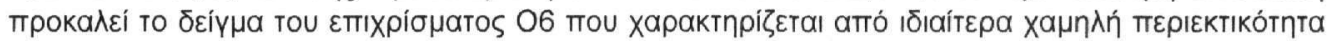

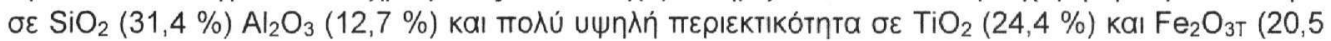

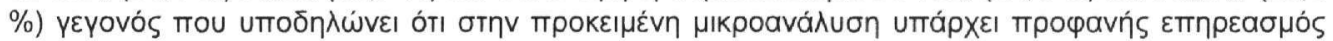

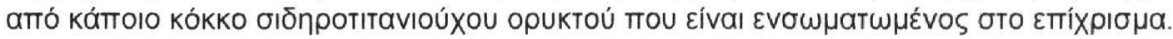

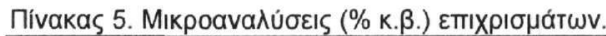

\begin{tabular}{|c|c|c|c|c|c|c|c|c|c|c|c|c|}
\hline Оотрако & $\mathrm{SiO}_{2}$ & $\mathrm{TiO}_{2}$ & $\mathrm{Al}_{2} \mathrm{O}_{3}$ & $\mathrm{Fe}_{2} \mathrm{O}_{3} \mathrm{~T}$ & $\mathrm{MgO}$ & $\mathrm{CaO}$ & $\mathrm{Na}_{2} \mathrm{O}$ & $\mathrm{K}_{2} \mathrm{O}$ & $\mathrm{P}_{2} \mathrm{O}_{5}$ & $\mathrm{PbO}$ & CuO & Eúvoגo \\
\hline $\mathrm{O} 2$ & 50,5 & 0,4 & 23,3 & 2,7 & 1,6 & 0,9 & 0,6 & 10,9 & 1,5 & 7,4 & - & 99,8 \\
\hline $\mathrm{O} 3$ & 51,6 & - & 29,6 & 1,5 & - & - & 2,3 & 12,8 & - & 2,0 & - & 99,8 \\
\hline O6 & 31,4 & 24,4 & 12,7 & 20,5 & 3,1 & 1,3 & 1,2 & 2,8 & - & 2,5 & - & 99,9 \\
\hline
\end{tabular}

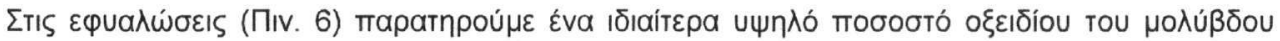

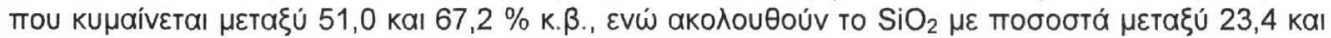

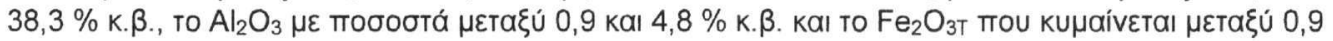

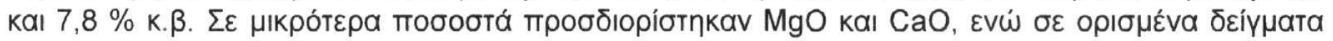
$\mathrm{Na}_{2} \mathrm{O}, \mathrm{K}_{2} \mathrm{O}, \mathrm{P}_{2} \mathrm{O}_{5} \mathrm{Kal} \mathrm{CuO}$.

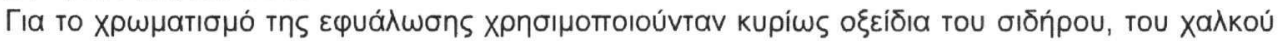

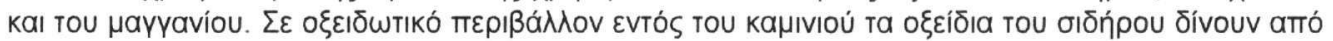

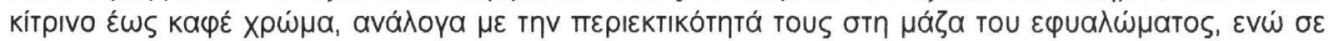

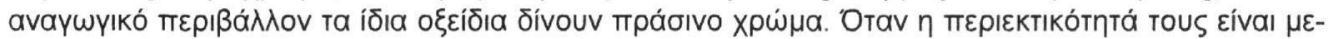

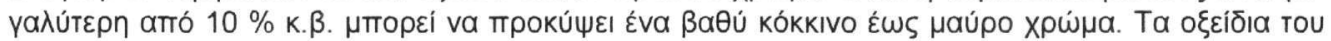

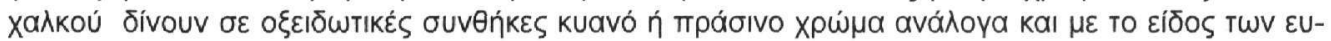

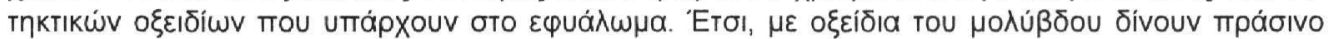

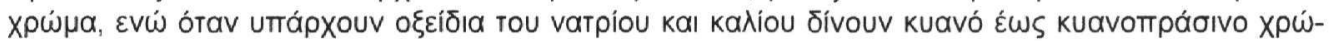

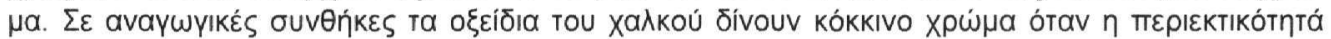

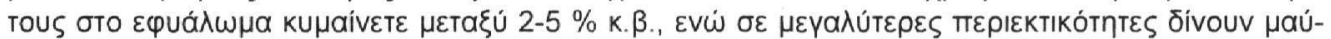

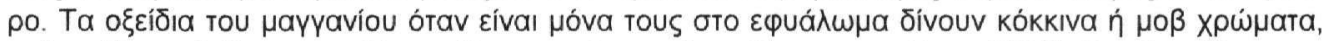

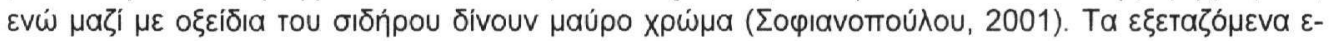

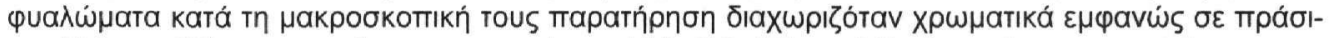

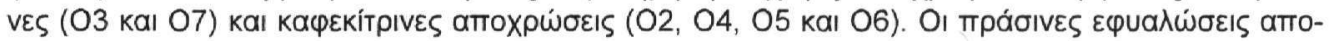

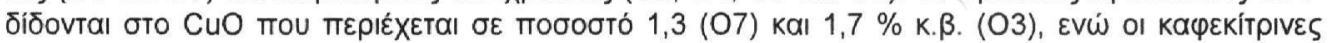

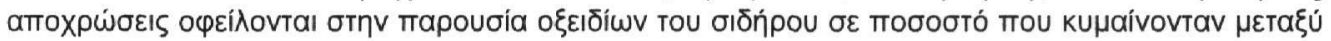

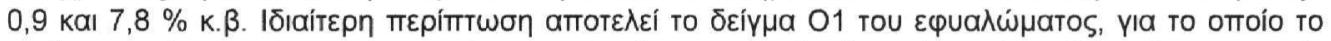

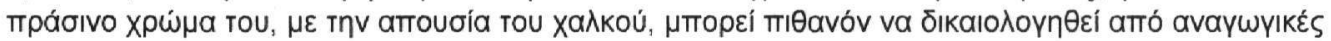

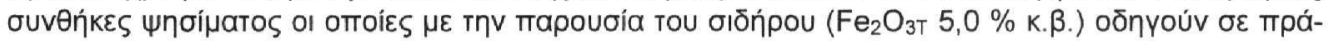

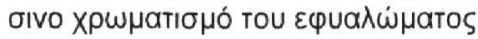

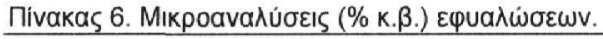

\begin{tabular}{ccccccccccccc}
\hline Oбтрако & $\mathrm{SiO}_{2}$ & $\mathrm{TiO}_{2}$ & $\mathrm{Al}_{2} \mathrm{O}_{3}$ & $\mathrm{Fe}_{2} \mathrm{O}_{3} \mathrm{~T}$ & $\mathbf{M g O}$ & $\mathrm{CaO}$ & $\mathrm{Na}_{2} \mathrm{O}$ & $\mathrm{K}_{2} \mathrm{O}$ & $\mathrm{P}_{2} \mathrm{O}_{5}$ & $\mathrm{PbO}$ & $\mathrm{CuO}$ & $\Sigma$ $u ́ v o \lambda o$ \\
\hline $\mathrm{O} 1$ & 36,1 & - & 4,5 & 5,0 & 0,4 & 0,7 & 0,9 & 1,2 & - & 51,0 & - & 99,8 \\
$\mathrm{O} 2$ & 38,3 & - & 4,4 & 0,9 & 0,2 & 1,3 & 0,7 & 1,1 & 0,5 & 52,3 & - & 99,7 \\
$\mathrm{O} 3$ & 32,1 & - & 2,7 & 1,4 & 0,5 & 1,3 & - & - & - & 60,2 & 1,7 & 99,9 \\
$\mathrm{O} 4$ & 26,1 & - & 1,3 & 5,3 & 0,7 & 4,8 & - & 0,4 & 0,8 & 60,5 & - & 99,9 \\
$\mathrm{O} 5$ & 25,0 & - & 4,8 & 2,5 & 1,0 & 1,4 & - & - & - & 65,1 & - & 99,8 \\
$\mathrm{O} 6$ & 25,8 & - & 0,9 & 7,8 & 0,4 & 5,9 & 0,6 & 0,5 & - & 58,0 & - & 99,9 \\
$\mathrm{O} 7$ & 23,4 & - & 3,7 & 1,3 & 0,5 & 1,2 & 0,6 & 0,7 & - & 67,2 & 1,3 & 99,9 \\
\hline
\end{tabular}

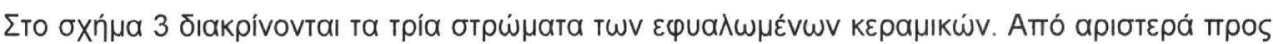

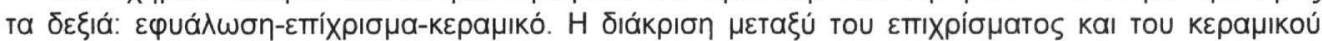

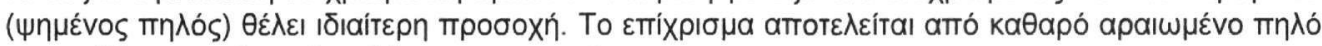

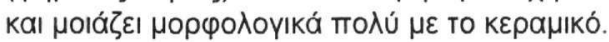

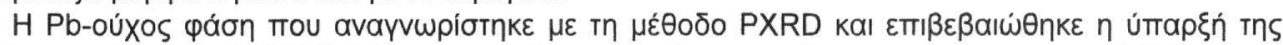

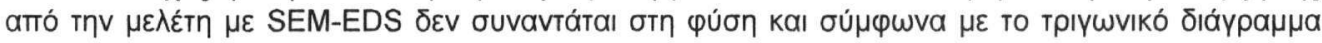

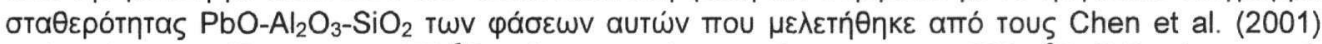

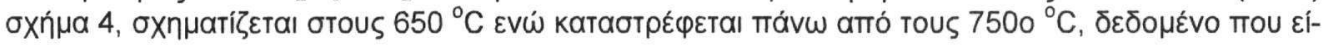




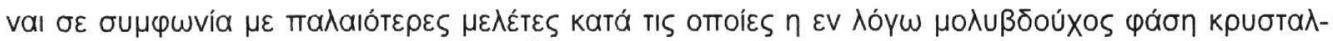

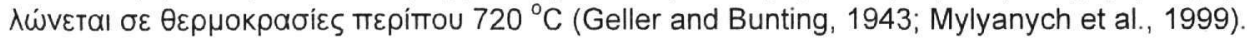

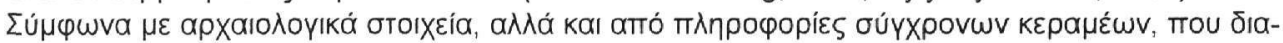

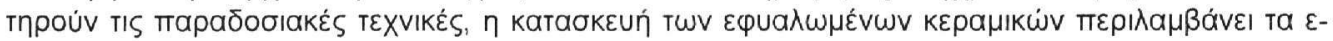

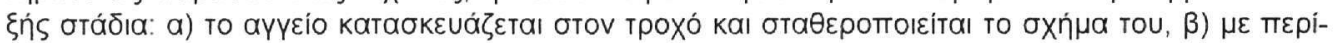

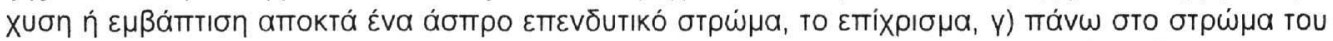

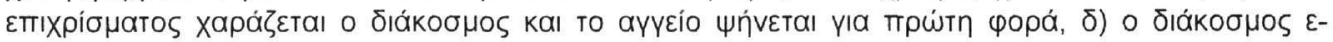

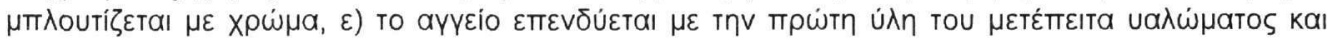

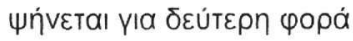

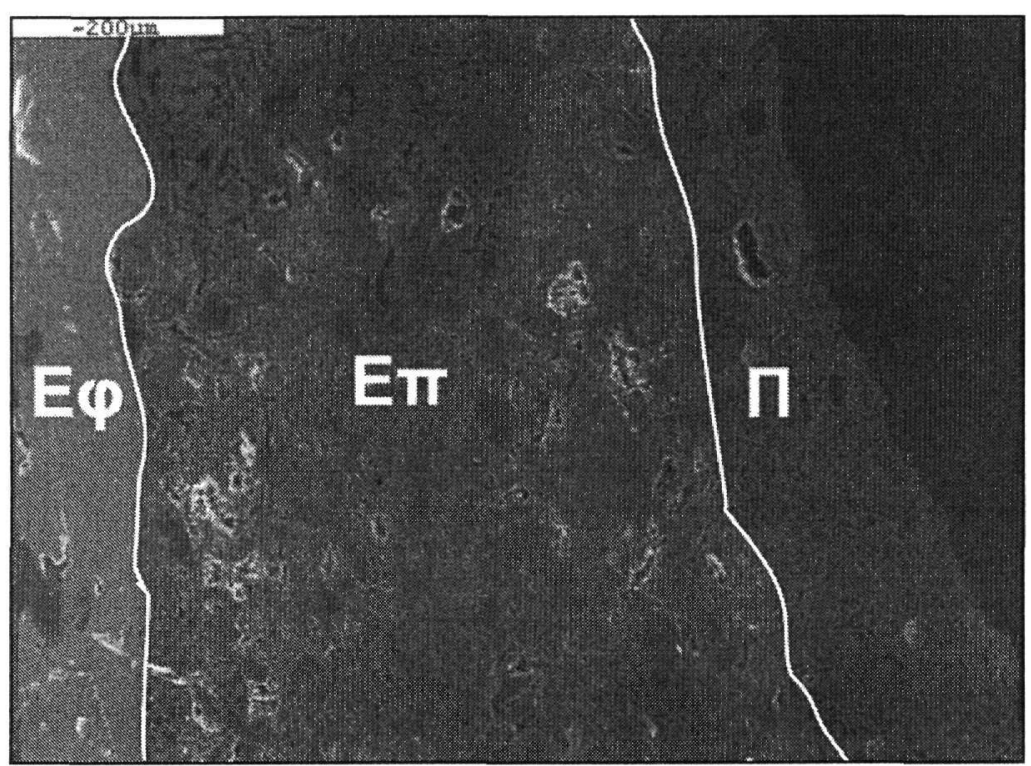

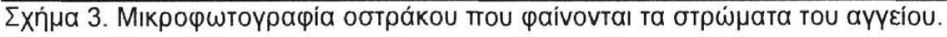

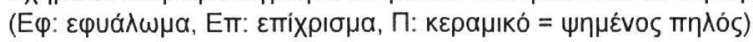

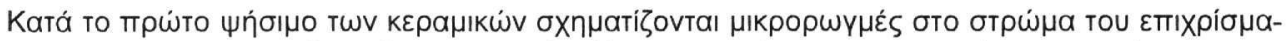

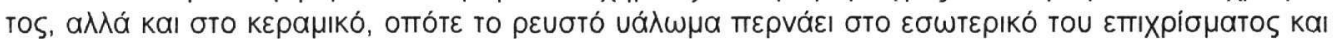

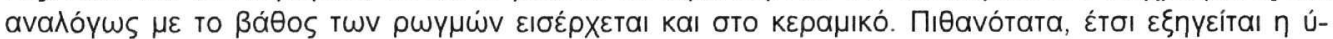

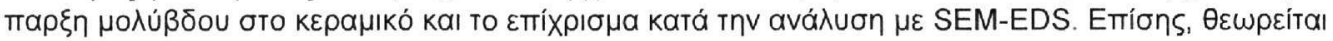

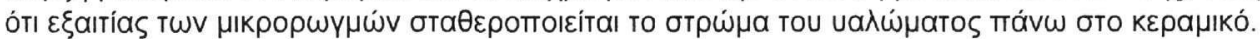

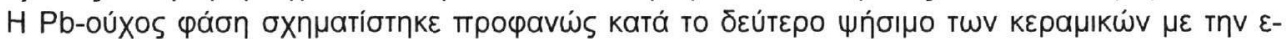

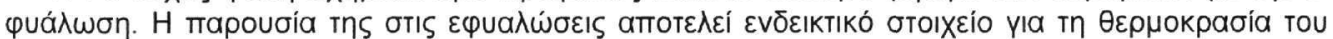

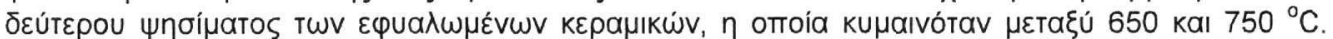

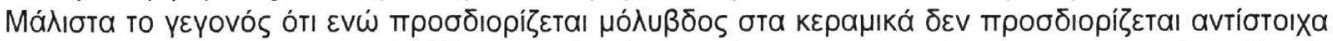

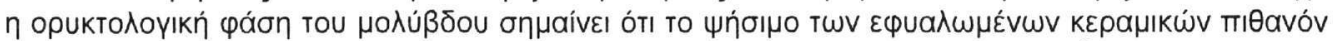

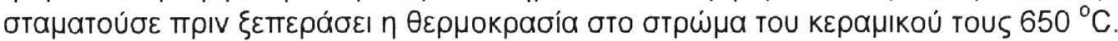

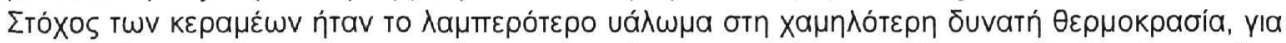

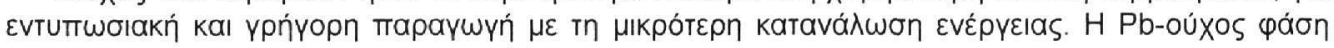

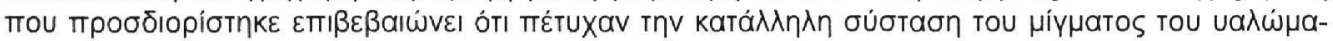

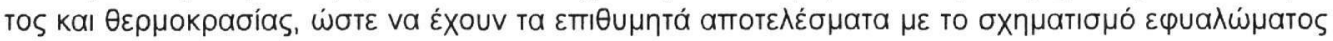

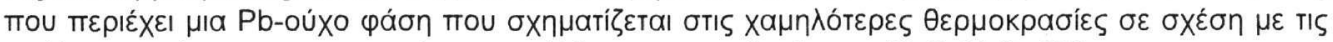

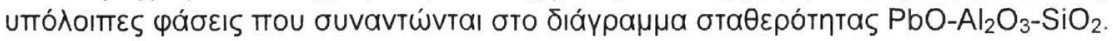

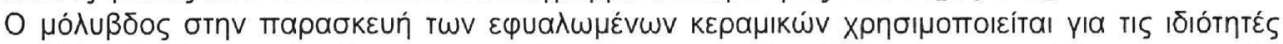

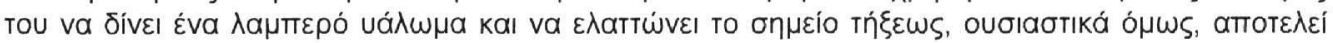

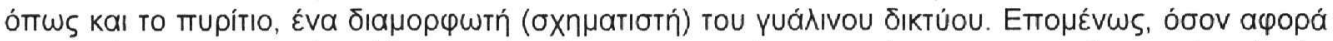

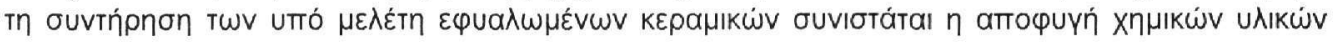




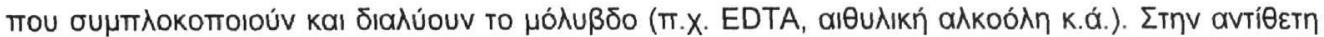

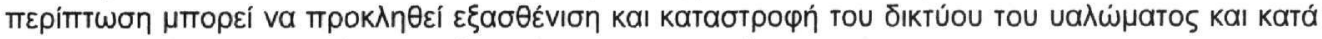

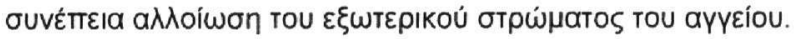

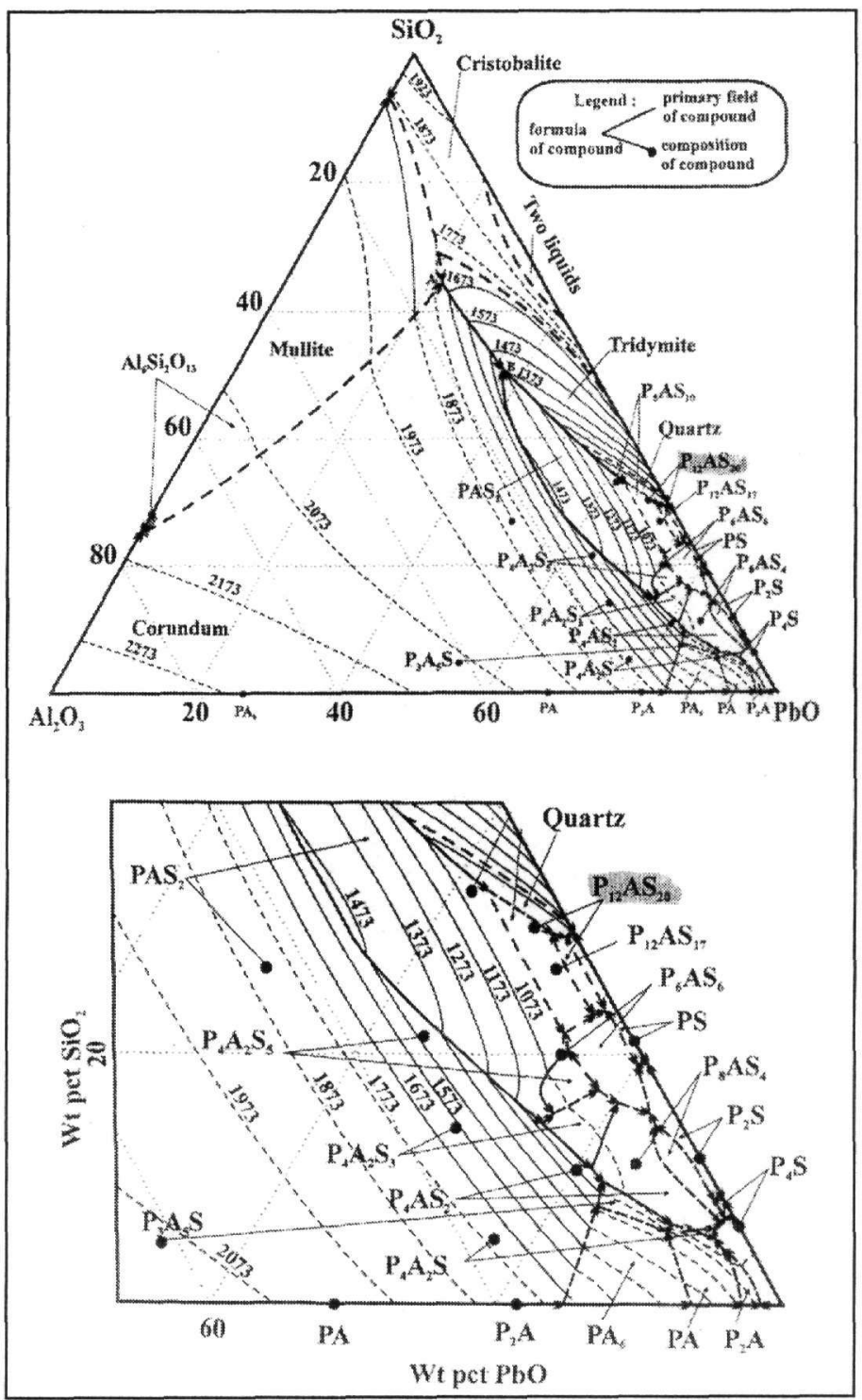

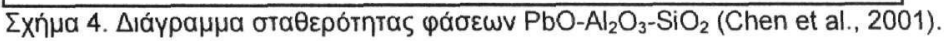

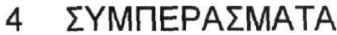

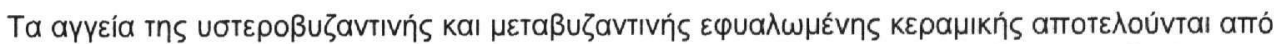

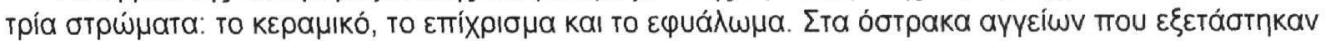

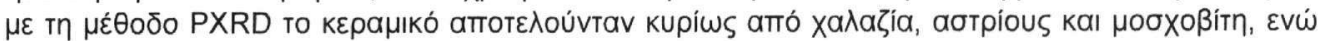

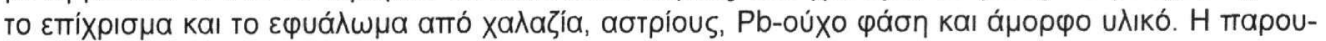

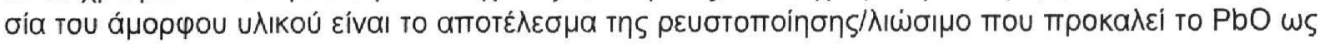

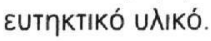




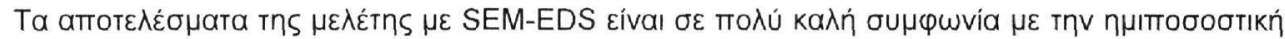

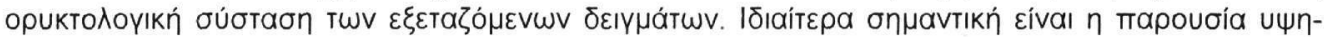

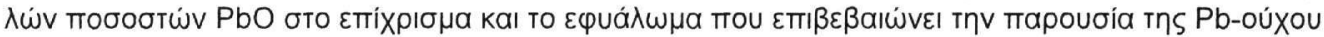

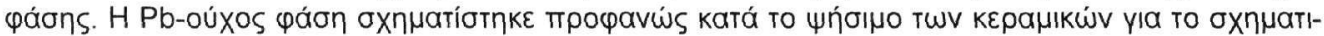

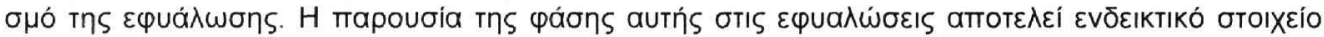

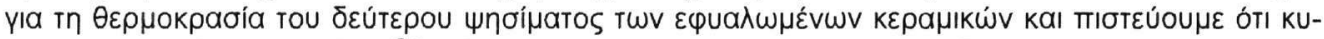

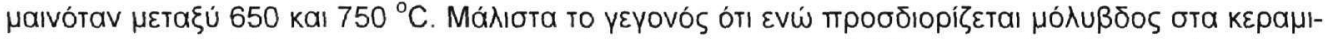

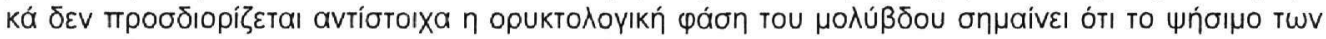

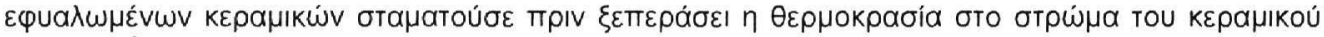
Tous $650^{\circ} \mathrm{C}$.

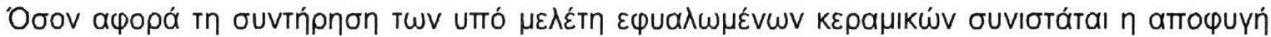

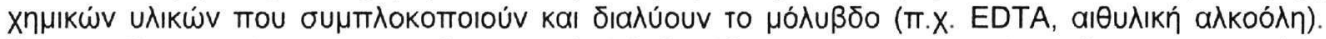

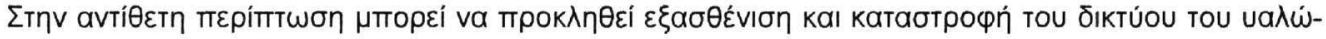

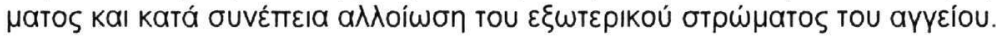

\section{ANAФOPE $\Sigma$}

Chen, S., Zhao, B., Hayes, P.C., and Jak, E., 2001. Experimental study of the phase equilibria in the PbO$\mathrm{Al}_{2} \mathrm{O}_{3}-\mathrm{SiO}_{2}$ system, Metallurgical and Materials Transactions $B$ 32B, 997-1005.

Deer, A.W., Howie, R.A., and Zussman, J., 1996. An introduction to the rock-forming minerals (2 ${ }^{\text {nd }}$ ed.). Essex, Longman, $696 \mathrm{p}$.

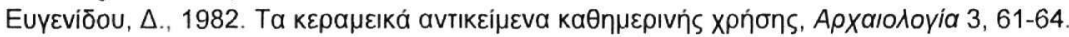

Geller, R., and Bunting, E., 1943. The system $\mathrm{PbO}-\mathrm{SiO}_{2}-\mathrm{Al}_{2} \mathrm{O}_{3}$. J. Nat. Bur. Stand. 31, 255-270.

Guinier, A., 1963. X-Ray diffraction in crystals, imperfect crystals and amorphous bodies, San Francisco, Freeman H.W. and Company, $378 \mathrm{p}$.

JCPDS-ICDD, 1997. PDF-2, Data Base (Sets 1-47), CD-ROM, Ver. 1.30.

Kantiranis, N., Tsirambides, A., Filippidis, A., and Christaras, B., 1999. Technological characteristics of the calcined limestone from Agios Panteleimonas, Macedonia, Greece. Materials \& Structures 32, 546-551.

Kantiranis, N., Tsiramdides, A., Filippidis, A., and Christaras, B., 1999. Technological characteristics of the calcined limestone from Agios Panteleimonas, Macedonia, Greece. Materials \& Structures 32, 546-551.

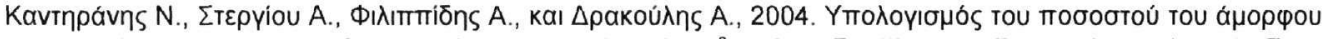

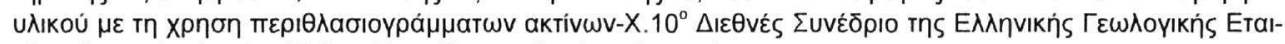

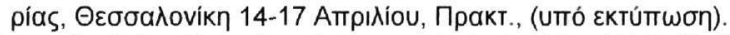

Mylyanych, O.A., Sheredko, A.M., and Melnyk K.S., 1999. Study of glass structures and crystalline phases in the $\mathrm{PbO}-\mathrm{Al}_{2} \mathrm{O}_{3}-\mathrm{SiO}_{2}$ system, Journal of Analytical Atomic Spectroscopy 14, 513-521.

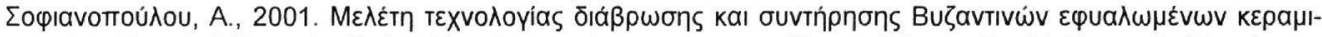

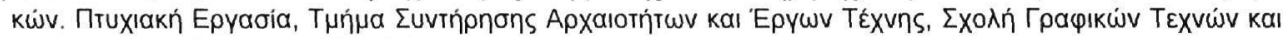

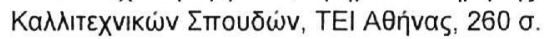

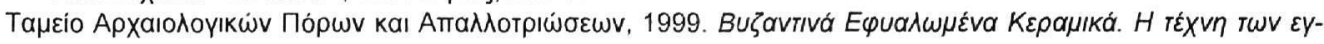

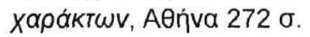




\section{ABSTRACT}

\section{STUDY OF TECHNOLOGY OF GLAZED POTTERY FROM AN EXCAVATION IN THESSALONIKI, GREECE}

Sofianopoulou A. ${ }^{1}$, Labropoulos B. ${ }^{2}$, and Kantiranis N. ${ }^{3}$

${ }^{1}$ Conservator of Antiquities and Works of Art, Ipsilantou 6, 54248 Thessaloniki, alecasof@otenet.gr

${ }^{2}$ Department of Conservation of Antiquities and Works of Art, TEl Athens, 12210 Athens, blabro@teiath.gr

${ }^{3}$ Faculty of Sciences, Aristotle University of Thessaloniki, 54124 Thessaloniki, kantira@geo.auth.gr

Samples of seven shells from vessels of glazed pottery of Palaeologan and post Byzantine period that were found during saving excavation of the 9th Ephoreia of Byzantine Antiquities in the centre of Thessaloniki, were studied with the methods of PXRD and SEM-EDS in order to investigate their firing conditions. Each shell is separated in three layers: the biscuit, the slip and the glaze. Biscuit constituted mainly of quartz, feldspars and muscovite, while the slip from quartz, feldspars, $\mathrm{Pb}$-rich phase and muscovite. The glaze is constituted mainly by the $\mathrm{Pb}$-rich phase that was recognized in the slip, quartz and feldspars. Amorphous phase was determined in all samples with increased percentages from biscuit to glaze. The EDS study confirms most of the results of PXRD study and shows, particularly in the glaze, the presence of very high percentages of $\mathrm{PbO}$. The presence of $\mathrm{Pb}$-rich phase in glaze constitutes an evidence for the temperature of second firing of glazed pottery and we believe that this temperature varied between 650 and $750{ }^{\circ} \mathrm{C}$. Relatively to the maintenance of the studied glazed ceramics is recommended the reject of chemicals that solvating and dissolving the lead (e.g. EDTA, ethyl alcohol). The use of these chemicals can be cause weakening and destruction of enamelling and accordingly alteration of exterior layer of vessel. 